\title{
Management of pericardial effusion by drainage: a survey of 10 years' experience in a city centre general hospital serving a multiracial population
}

\author{
Christopher R Gibbs, Robert D S Watson, Shyam P Singh, Gregory Y H Lip
}

\begin{abstract}
The aim of the study was to determine the aetiology of large and symptomatic pericardial effusions and to review the management and subsequent outcome. A survey was done on a consecutive cases of patients who had undergone percutaneous pericardiocentesis over a 10 year period in a city centre general hospital serving a multiethnic catchment population. In all, 46 patients ( 24 male, 22 female; age range 16 to 90 years, mean 54 years) underwent a total of 51 pericardial drainage procedures (or attempted pericardiocentesis) between 1989 and 1998. Malignancy (44\%), tuberculosis (26\%), idiopathic (11\%), and post-cardiac surgery $(9 \%)$ were the most common causes of pericardial effusion. The most common presenting symptoms were breathlessness $(90 \%)$, chest pain $(74 \%)$, cough $(70 \%)$, abdominal pain $(61 \%)$ (presumed to be related to hepatic congestion), and unexplained fever ( $28 \%$ ). In the 12 cases of tuberculous pericarditis, nine occurred in patients of Indo-Asian origin, and three in patients of AfroCaribbean origin. Fever, night sweats, and weight loss were common among these patients, occurring in over $80 \%$ of cases of tuberculous pericarditis. Pulsus paradoxus was the most specific sign $(\mathbf{1 0 0 \%})$ for the presence of echocardiographic features of tamponade, with strongest positive predictive value $(100 \%)$. Although malignancy remains the most common cause in developed countries, tuberculous disease should be considered in patients from areas where tuberculosis is endemic. Percutaneous pericardiocentesis remains an effective measure for the immediate relief of symptoms in patients with cardiac tamponade, although its diagnostic yield in tuberculous pericarditis is relatively low.

(Postgrad Med f 2000;76:809-813)
\end{abstract}

Keywords: tuberculosis; pericardial effusions; percutaneous pericardiocentesis

Patients with pericardial effusions present to all medical specialties, although their care is often supervised by cardiologists. Patients with symptomatic effusions can be severely unwell at presentation, and the immediate aim must be the relief of symptoms, although secondary aims in these patients should include determination of the cause of the effusion and preventing recurrence.

Percutaneous needle pericardiocentesis remains the most common therapeutic procedure for the early management of symptomatic effusions and it continues to be used as a diagnostic procedure in some patients with asymptomatic pericardial effusions. However, pericardiocentesis is itself associated with morbidity and mortality, and there is limited information about the diagnostic role and outcomes of percutaneous pericardial drainage, especially in some groups of patients-for example, those with large tuberculous effusions.

Over the last 10 years we have maintained a prospective register of consecutive cases of patients who have undergone percutaneous pericardiocentesis in our city centre general hospital, which serves a multiethnic catchment population. The aim of this register has been to determine the aetiology of large and symptomatic pericardial effusions and to review the management and subsequent outcome of these patients.

\section{Methods}

Consecutive patients who underwent pericardiocentesis or attempted pericardiocentesis at our hospital between 1989 and 1998 inclusive were included in the database. City Hospital is a 770 bedded hospital with three consultant cardiologists and a wide range of general medical services, although there are no on site facilities for routine renal dialysis. The local urban catchment population of approximately 300000 people is ethnically diverse, and the 1991 census of west Birmingham indicated that in the age band $30-65$ years, $22 \%$ of residents were Indo-Asian, $66 \%$ white, and $12 \%$ Afro-Caribbean.

Features documented for each patient included the following:

- presenting symptoms;

- physical signs:

-tachycardia ( $>100$ beats/min) and the presence of an arrhythmia (for example, atrial fibrillation);

-hypotension (systolic blood pressure $<100$ $\mathrm{mm} \mathrm{Hg}$ );

-pulsus paradoxus (> $10 \mathrm{~mm} \mathrm{Hg}$ difference between systolic blood pressure during inspiration and expiration); 
Table 1 Aetiology of pericardial effusions managed with percutaneous pericardiocentesis

\begin{tabular}{|c|c|c|c|c|}
\hline \multirow[b]{2}{*}{ Diagnosis } & \multirow{2}{*}{$\begin{array}{l}\%(n) \\
\text { Total }\end{array}$} & \multicolumn{3}{|c|}{ Ethnicity (n) } \\
\hline & & White & Black & Asian \\
\hline Malignancy & $44(20)$ & 17 & 2 & 1 \\
\hline Tuberculosis & $26(12)$ & - & 3 & 9 \\
\hline Idiopathic & $11(5)$ & 5 & - & - \\
\hline Post-cardiac surgery & $9(4)$ & 3 & 1 & - \\
\hline Post-MI & $4(2)$ & 2 & - & - \\
\hline Autoimmune (SLE, rheumatoid arthritis) & $4(2)$ & 2 & - & - \\
\hline Viral & $2(1)$ & 1 & - & - \\
\hline
\end{tabular}

MI, myocardial infarction; SLE, systemic lupus erythematosus.

-raised jugular venous pressure $(>3 \mathrm{~cm})$; -abnormal cardiac auscultation (diminished heart sounds, pericardial rub);

- results of baseline investigations:

-ECG (small complexes, electrical alternans);

-chest $x$ ray;

-echocardiographic features documenting size of effusion ( $M$ mode dimension anteriorly and/or posteriorly $>2 \mathrm{~cm}=\mathrm{a}$ large effusion) and evidence of cardiac tamponade, defined as the presence of cardiac chamber collapse during diastole;

- the primary indication for pericardiocentesis:

- cardiac tamponade (on clinical and/or echocardiographic criteria)

-symptomatic pericardial effusion, without evidence of cardiac tamponade

-diagnostic (asymptomatic pericardial effusion)

- aetiology of pericardial effusion;

- details of procedure, including route, volume of fluid drained, subsequent investigations and complications;

- short and long term outcomes.

Data were entered onto a spreadsheet for analysis.

\section{Results}

PATIENT CHARACTERISTICS

In all, 46 patients (24 male, 22 female; age range 16 to 90 years, mean 54) underwent a total of 51 pericardial drainage procedures (or attempted pericardiocentesis) between 1989 and 1998. These included 30 white, 10 Indo-Asian, and six Afro-Caribbean patients.

AETIOLOGY

The underlying causes of the pericardial effusions are outlined in table 1. Malignancy $(44 \%)$, tuberculosis (26\%), idiopathic (11\%), and post-cardiac surgery $(9 \%)$ were the most common causes. The primary sites of the malignant cases are outlined in table 2. The lung and breast were the most common primary sites, although the origin of the malignancy could not be identified in all patients, even following necropsy.

\section{CLINICAL FEATURES}

The duration of onset of the symptoms was extremely variable (median 14 days, range 4 days to 10 months). The most common presenting symptoms were breathlessness (90\%), chest pain (74\%), cough $(70 \%)$, abdominal pain $(61 \%)$ (presumed to be related to hepatic congestion), and unexplained fever
Table 2 Primary site of malignant pericardial effusions

\begin{tabular}{ll}
\hline Malignancy & $\%(n)$ \\
\hline Breast & $25(5)$ \\
Lung (adenocarcinoma) & $20(4)$ \\
Lung (squamous cell carcinoma) & $10(2)$ \\
Adenocarcinoma (unknown primary) & $10(2)$ \\
Non-Hodgkin's lymphoma & $10(2)$ \\
Ovarian & $5(1)$ \\
Thyroid & $5(1)$ \\
Nasopharyngeal carcinoma & $5(1)$ \\
Mesothelioma & $5(1)$ \\
Chronic lymphocytic leukaemia & $5(1)$ \\
\hline
\end{tabular}

$(28 \%)$. In the 12 cases of tuberculous pericarditis, nine occurred in patients of Indo-Asian origin and three in patients of Afro-Caribbean origin. Fever, night sweats, and weight loss were common among these patients, occurring in over $80 \%$ of those with tuberculous pericarditis.

PHYSICAL SIGNS

Cardiac tamponade was the primary indication for pericardiocentesis in the majority of the cases $(79 \% ; n=40)$. Drainage was also performed in $12 \%$ of patients $(n=6)$ with symptomatic effusions, but without clinical or echocardiographic evidence of tamponade. Nine per cent $(n=5)$ were performed for diagnostic purposes in patients with asymptomatic pericardial effusions. In the cases of tuberculous effusion, five patients presented with tamponade, four with large symptomatic effusions, and three were asymptomatic. In the latter, pericardiocentesis was performed as part of a diagnostic screen.

In terms of the clinical signs, a tachycardia, raised jugular venous pressure, and the presence of pulsus paradoxus were all strongly associated with echocardiographic evidence of cardiac tamponade (table 3). Atrial fibrillation was documented in five patients, and the presence of atrial fibrillation was considered to be related to the pericardial disease in four of these. Pulsus paradoxus was the most specific sign $(100 \%)$ for the presence of echocardiographic features of tamponade, with strongest positive predictive value $(100 \%)$. However, although the presence of these physical signs is an important diagnostic finding, their negative predictive value is limited and the absence of one or all of these signs does not exclude the presence of cardiac tamponade. Importantly, hypotension was an uncommon finding in patients with echocardiographic evidence of tamponade, occurring in only $24 \%$ of cases $(n=11)$.

\section{INVESTIGATIONS}

Radiographic cardiomegaly was present in all patients who underwent pericardiocentesis, although small complexes on the ECG were only present in $39 \%(n=18)$ of patients who presented with tamponade. Clear evidence of electrical alternans was present in one patient (with a tuberculous effusion and evidence of tamponade).

Mitotic cells were present in the pericardial fluid in $60 \%(n=12)$ of samples from patients with effusions related to malignant diseasepredominantly from breast, lung and adeno- 
Table 3 Sensitivity, specificity, and positive and negative predictive value of physical signs in patients with echocardiographic evidence of cardiac tamponade

\begin{tabular}{|c|c|c|c|c|c|}
\hline Physical sign & Present \% (n) & Sensitivity (\%) & Specificity (\%) & $\begin{array}{l}\text { Positive } \\
\text { predictive value } \\
(\%)\end{array}$ & $\begin{array}{l}\text { Negative } \\
\text { predictive value } \\
(\%)\end{array}$ \\
\hline Tachycardia ( $>100$ beats/min) & $88(40)$ & 88 & 67 & 92 & 57 \\
\hline Raised jugular venous pressure $(>3 \mathrm{~cm})$ & $88(40)$ & 88 & 83 & 96 & 62 \\
\hline Pulsus paradoxus $(>10 \mathrm{~mm} \mathrm{Hg})$ & $80(37)$ & 80 & 100 & 100 & 55 \\
\hline Hypotension (systolic BP $<100 \mathrm{~mm} \mathrm{Hg}$ ) & $24(11)$ & 24 & 83 & 85 & 21 \\
\hline Diminished heart sounds & $24(11)$ & 24 & 100 & 100 & 11 \\
\hline
\end{tabular}

*Atrial fibrillation was documented in 5 patients and the presence of atrial fibrillation was considered to be related to the pericardial disease in 4 of these patients.

carcinoma of unknown primary. Biochemical analysis of samples (protein, lactate dehydrogenase) did not significantly aid the diagnosis in any of these patients.

Tuberculous effusions were lymphocytic in $75 \%$ of patients $(n=9)$, and acid fast bacilli were present on direct smear examination of the pericardial fluid in only $17 \%$ of cases $(n=2)$, with subsequent positive culture in $25 \%(n=3)$.

\section{PROCEDURE AND COMPLICATIONS}

Successful pericardiocentesis was performed in 48 cases, with failed pericardiocentesis in three. The xiphisternal approach (with ECG monitoring) was successful as the first line approach in $92 \%$ of cases $(n=44)$, with the left parasternal as the first line approach in two. The left parasternal approach proved successful following unsuccessful attempts at xiphisternal pericardiocentesis in two patients. Two patients underwent urgent surgical pericardiotomy when drainage could not be secured by either the xiphisternal or the parasternal route. In all cases, a pericardial drain was left in situ (for a median of 48 hours) and an average of $650 \mathrm{ml}$ (range 150 to $1250 \mathrm{ml}$ ) of pericardial fluid was drained during each successful procedure. More than $90 \%(n=42)$ of the effusions were haemorrhagic, irrespective of the underlying aetiology.

Complications were documented in $15 \%$ of the procedures $(n=6)$. Complications included right ventricular puncture $(n=4)$, secondary pericardial infection requiring antibiotic treatment and prolonged inpatient stay $(n=2)$, and a severe vasovagal episode $(n=1)$. None of the episodes of right ventricular puncture was associated with the development of significant cardiac tamponade or the requirement for surgical drainage. There were no procedure related fatalities.

SUBSEQUENT COURSE AND OUTCOME

After a mean follow up of 16 months, $45 \%$ of the patients $(n=21)$ had died as a result of complications related to the underlying disease or from other illnesses, although there were no deaths directly attributable to pericardial disease. One patient with a malignancy related effusion developed recurrent tamponade within four weeks of the original drainage procedure, but was treated successfully with closed balloon pericardiotomy.

All patients with tuberculous effusions were initially treated with oral steroids (for six to eight weeks) and six months of conventional antituberculous treatment, although one patient (with a history of tuberculosis) was treated for an extended period (18 months). In all, nine of the patients with tuberculous pericarditis had recovered without clinical or echocardiographic evidence of recurrent pericardial effusions after a mean follow up of 32 months (range 4 to 64 months). However, two patients developed recurrent effusion within four weeks of the original pericardiocentesis, and both were managed with urgent surgical pericardiotomy. There were no recorded cases of effusive constriction, but there was one case of chronic pericardial constriction occurring in a patient who had presented with a 10 month history of symptoms.

\section{Discussion}

In the Western world malignancy is the most common cause of large pericardial effusions, followed by uraemia. ${ }^{12}$ In the present series, the absence of on site specialised renal services (including dialysis) almost certainly explains the relatively low incidence of uraemic pericardial effusions. However, our series has a high incidence of large tuberculous pericardial effusions when compared with previously published European case series, and this is a reflection of the local multicultural population, with a high proportion of Indo-Asian and AfroCaribbeans.

Our series confirms that cardiac tamponade is the most common indication for pericardiocentesis and, indeed, that therapeutic pericardiocentesis is mandatory in patients with cardiac tamponade. A large effusion with a circumferential echo space of $>1 \mathrm{~cm}$ anteriorly or posteriorly is reported to be a powerful predictor of the development of tamponade. ${ }^{3}$ Nevertheless, cardiac tamponade is usually a clinical diagnosis which is confirmed by echocardiography, where right ventricular (or right atrial) collapse is usually observed ${ }^{4-7}$; however, left ventricular collapse is also seen, especially in the more advanced stages of tamponade $^{8}$ and following cardiac surgery. ${ }^{9}$ Rarely, despite life threatening tamponade, right atrial or right ventricular diastolic collapse may not be present in some patients, particularly following cardiac surgery. ${ }^{10}$ Indeed, the volume of fluid required to produce cardiac tamponade is also variable and is dependent on both the rate of accumulation and the distensibility of the pericardial sac.

In our series, pulsus paradoxus was the single most reliable physical cardiovascular sign of tamponade and this is compatible with other case series. ${ }^{11}$ However, clinical signs are not entirely reliable, as a paradoxical arterial pulse 
is not specific for tamponade and may be absent in several situations, ${ }^{12}$ for example, in the presence of regional right atrial compression, ${ }^{13}$ with regional tamponade of the left heart (as seen following cardiac surgical procedures), and in patients with left ventricular dysfunction. ${ }^{14}$ Hypotension, diminished heart sounds, and a pericardial rub were often absent in our series, and although valuable when present, the absence of these signs is of limited diagnostic value.

Pericardial aspiration is not indicated as a routine investigation, because of the low diagnostic yield, ${ }^{15}$ although, in addition to cardiac tamponade, a clinical suspicion of purulent pericarditis is an indication for pericardiocentesis. Percutaneous pericardiocentesis was associated with initial relief of symptoms in almost all cases, and when the drain was left in situ for 48 hours the recurrence rate was generally low, although leaving the drain in situ increases the risk of subsequent infection. Although sclerotherapy with tetracycline hydrochloride or bleomycin sulphate has been used to reduce the recurrence rate, ${ }^{16}$ this therapeutic option was not routinely employed in our case series. Subxiphoid pericardiotomy is another option in cases of recurrence: this procedure may be performed under local anaesthesia, and has a low morbidity and mortality. ${ }^{17}$

Tuberculosis remains a common cause of pericarditis in developing countries, although it accounts for less that $5 \%$ of cases in the Western world. ${ }^{18}$ Although the presentation of tuberculous pericarditis is variable, right upper abdominal discomfort caused by liver congestion is reported to be common in these patients. ${ }^{19}{ }^{20}$ In our series, fever, night sweats and weight loss were common, as observed in other case series. ${ }^{21}{ }^{22}$ In more than $80 \%$ of cases the aspirated pericardial fluid in tuberculosis is haemorrhagic. ${ }^{23}$ The diagnosis of tuberculous pericarditis is confirmed by the presence of acid fast bacilli in the pericardial fluid or on biopsy of the pericardium. As seen in our series, acid fast bacilli are difficult to isolate from pericardial fluid ${ }^{24}$; they are rarely seen on direct examination, and the positive culture rate from conventional culture is only around $50 \%$, although immediate inoculation in double strength liquid Kirchner culture medium increases the yield to $75 \% .^{25}$ The diagnostic yield of pericardiocentesis and pericardial biopsy also appears to be similar. A "probable" diagnosis of tuberculous pericarditis is thus made by the confirmation of tuberculosis elsewhere in a patient with an unexplained pericardial effusion, and a "definite or probable" diagnosis is made in up to $70 \%$ of patients treated for tuberculous pericarditis. ${ }^{26}{ }^{27}$ The chest $x$ ray shows active tuberculosis in only $30 \%$ of patients with tuberculous pericarditis, and a pleural effusion is present in $40-60 \%$ of cases. ${ }^{27-29}$ The tuberculin skin test is generally of limited value in patients from developing countries, because of the high prevalence of primary tuberculosis and the widespread use of BCG immunisations. ${ }^{29}$

In our series, four of the patients were treated without microbiological or other con- firmation of tuberculosis. These patients were all from endemic areas (India, Pakistan, Bangladesh, Jamaica), and the clinical history (fever, night sweats, and weight loss), the presence of a lymphocytic pericardial effusion, and an adequate response to empirical antituberculous treatment were highly suggestive of tuberculous pericarditis. Empirical treatment has been recommended in patients from endemic areas, where investigations fail to yield a diagnosis, although there is no evidence supporting empirical treatment in patients from non-endemic areas. ${ }^{30}$ Conventional antituberculous treatment has dramatically improved the prognosis in tuberculous pericarditis. In one large scale prospective South African study, oral prednisolone appeared to reduce the risk of reaccumulation of pericardial fluid, and the requirement for repeat pericardiocentesis, with a trend towards reduced mortality. ${ }^{31}$ Furthermore, the use of prednisolone in patients with established pericardial constriction was associated with a (non-significantly) lower mortality from pericarditis and a reduced requirement for pericardectomy. ${ }^{32}$ Although the use of steroids remains controversial, ${ }^{33}$ our practice is still to use adjuvant prednisolone in all patients treated with antituberculous chemotherapy for tuberculous pericarditis.

In other published case series, the complication rates of percutaneous pericardiocentesis range from $4 \%$ to $40 \%$, with an overall estimated procedure related mortality rate of $2 \%,{ }^{34}$ although echocardiographically guided aspiration has been reported to be associated with lower complication rates. ${ }^{35} 36$ Myocardial puncture is the commonest reported complication in most case series, with a frequency of $2-7 \%$, and our series reported right ventricular puncture in $8 \%$ of attempts at percutaneous pericardiocentesis. Right ventricular puncture is rarely a fatal complication, although severe cardiac tamponade, requiring surgical pericardiotomy, still occurs in a small proportion of cases. Other recognised complications include atrial and ventricular arrhythmias, ${ }^{37}$ severe vasovagal episodes, and pneumothoraces (which are rare and normally associated with the apical approach). The presence of effusion loculation reduces the likelihood of successful pericardiocentesis. $^{38}$

This study is based on the experience of a single centre and several operators were involved in performing the percutaneous procedures, although all were performed or supervised by experienced operators. A further limitation is the relatively short follow up, to date, of some of the patients with tuberculous pericarditis, in view of the fact that long term complications-particularly constrictive pericarditis - may only become evident many years after the original infection.

\section{CONCLUSIONS}

Percutaneous pericardiocentesis remains an effective measure for the immediate relief of symptoms in patients with cardiac tamponade. Nevertheless, its diagnostic yield in tuberculous pericarditis is relatively low. Percutaneous pericardiocentesis is associated with complica- 
tions in a small but important proportion of patients. While malignancy remains the most common cause in developed countries, tuberculous disease should be considered in patients from areas where tuberculosis is endemic.

1 Wilkes JD, Fidias P, Vaickus L, et al. Malignancy-related pericardial effusion. Cancer 1995:76:1377-87.

2 Corey RG, Campbell PT, van Trigt P, et al. Etiology of large pericardial effusions. Am 7 Med 1993;95:209-13.

3 Eisenberg MJ, Dunn MM, Kanth N, et al. Prognostic value of echocardiography in hospitalised patients with pericardial of echocardiography in hospitalised patients

4 Kronzon I, Cohen ML, Winer HE. Diastolic atrial Kronzon I, Cohen ML, Winer HE. Diastolic atrial compression: a sensitive echocardiographic

5 Engel PJ, Hon H, Fowler NO, et al. Echocardiographic study of right ventricular wall motion in cardiac tamponade. Am $\mathcal{F}$ Cardiol 1982;50:1018-21.

6 Singh S, Wann L, Schuchard, et al. Right ventricular and right atrial collapse in patients with cardiac tamponade: a combined echocardiographic and haemodynamic study. Circulation 1984;70:966-71.

7 Leimgruber P, Klopfenstein H, Wann L, et al. The haemodynamic derangement associated with right ventricular diastolic collapse in cardiac tamponade: an experimental echocardiographic study. Circulation 1983;68:612-20.

8 Chuttani K, Pandian NG, Mohanty PK, et al. Left ventricular diastolic collapse: an echocardiographic sign of regional cardiac tamponade. Circulation 1991;83:1999-2006.

9 Fyke FE, Tancredi RG, Shub C, et al. Detection of Fyke FE, Tancredi RG, Shub C, et al. Detection of
intrapericardial haematoma after open heart surgery: the intrapericardial haematoma after open heart surgery: the roles of echocardiography and
Coll Cardiol 1985;5:1496-9.

10 Chuttani K, Tischler MD, Pandian MG, et al. Diagnosis of cardiac tamponade after cardiac surgery: relative value of clinical, echocardiographic and haemodynamic signs. $A m$ Heart f 1994;127:913-18.

11 Guberman B, Fowler N, Engel P, et al. Cardiac tamponade in medical patients. Circulation 1981;64:633-40.

12 Fowler NO. Cardiac tamponade: a clinical or an echocardiographic diagnosis? Circulation 1993;87:1738-41.

13 Pepi M, Muratori M, Barbier P, et al. Pericardial effusion after cardiac surgery: incidence, site, size and haemodynamic consequences. Br Heart f 1994;72:327-31.

14 Hoit BD, Gabel M, Fowler NO. Cardiac tamponade in left ventricular dysfunction. Circulation 1990;82:1370-6.

15 Merce J, Sagrista-Sauleda J, Permanyer-Miralda G, et al. Should pericardial drainage be performed routinely in patients who have a large pericardial effusion without patients who have a large pericardial
tamponade? Am $\mathcal{M}$ Med 1998;105:106-9.

16 Vaitkus PT, Herrmann HC, LeWinter MM. Treatment of malignant pericardial effusion. $¥ A M A \quad 1994 ; 272: 59-64$

17 Alcan KE, Zabetakis PM, Marino ND, et al. Management of acute cardiac tamponade by subxiphoid pericardiotomy. ҒAMA 1982;247:1143-8

18 Permanyer-Miralda G, Sagrista-Sauleda J, Soler-Soler J. Primary acute pericardial disease: a prospective series of 231 consecutive patients. Am f Cardiol 1985;56:623-9.
19 Schirire V. Experience with pericarditis of Gruute Schuur Hospital, Cape Town: an analysis of one hundred and sixty cases over a six-year period. $S$ Afr Med f 1959;33:810-17.

20 Strang JIG. Tuberculous pericarditis. Clin Cardiol 1984;7: 667-70.

21 Hageman JH, d'Esopo ND, Glenn WWL. Tuberculosis of the pericardium: a long-term analysis of forty-four proved cases. N Engl f Med 1964;270:327-32.

22 Quale JM, Lipschik GY, Heurich AE. Management of tuberculous pericarditis. Ann Thorac Surg 1987;43:653-5.

23 Nugue O, Millaire A, Porte H, et al. Pericardioscopy in the etiologic diagnosis of pericardial effusion in 141 consecutive patients. Circulation 1996;94:1635-41.

24 Fowler NO, Manitsas GT. Infectious pericarditis. Prog Cardiovasc Dis 1973;16:323-36.

25 Strang G, Latouf S, Commerford P, et al. Bedside culture to confirm tuberculous pericarditis. Lancet 1991;338:1600-1.

26 Sagrista-Sauleda J, Permanyer-Miralda G, Soler-Soler J. Tuberculous pericarditis: ten-year experience with a prospective protocol for diagnosis and treatment. $7 \mathrm{Am}$ Coll Cardiol 1988;11:724-8.

27 Fowler NO. Tuberculous pericarditis. FAMA 1991;266:99103

28 Desai HN. Tuberculous pericarditis: a review of 100 cases. $S$ Afr Med F 1979;55:877-80.

$29 \mathrm{Ng}$ TTC, Strang JIG, Wilkins EGL. Serodiagnosis of pericardial tuberculosis. $Q \mathcal{F}$ Med 1995;88:317-20.

30 Permanyer-Miralda G, Sagrista-Sauleda J, Shebatai R, et al. Acute pericardial disease: an approach to etiologic diagnosis and treatment. In: Soler-Soler J, et al, eds. Pericardial disease: new insights and old dilemmas. Dordrecht: Kluwer, 1990.

31 Strang JIG, Kazaka HHS, Gibson DG, et al. Controlled clinical trial of complete open surgical drainage and of prednisolone in treatment of tuberculous pericardial effusion in Transkei. Lancet 1988;ii:759-64.

32 Strang JIG, Kazaka HHS, Gibson DG, et al. Controlled trial of prednisolone as adjuvant in treatment of tuberculous constrictive pericarditis in Transkei. Lancet 1987;ii:141822.

33 Lorell BH. Pericardial diseases. In: Braunwald E, ed. Heart disease: a textbook of cardiovascular medicine. Philadelphia: WB Saunders, 1997.

34 Ball JB, Morrison WL. Cardiac tamponade. Postgrad Med $\mathcal{F}$ 1997;73:141-5.

35 Callahan JA, Seward JB, Nishimura RA, et al. Twodimensional echo-cardiographically guided pericardiocentesis: experience in 117 consecutive cases. Am 7 Cardiol 1985;55:476-9.

36 Kopecky SL, Callahan JA, Tajik AJ, et al. Percutaneous pericardial catheter drainage: report of 42 consecutive cases. $\mathrm{Am}$ 7 Cardiol 1986;58:633-5.

37 Kwasnik EM, Koster JK, Lazarus JM, et al. Conservative management of uraemic pericardial effusions. F Thorac Cardiovasc Surg 1978;76:629-32.

38 Cooper JP, Oliver RM, Currie P, et al. How do the clinical findings in patients with pericardial effusions influence the success of aspiration? Br Heart f 1995;73:351-4. 